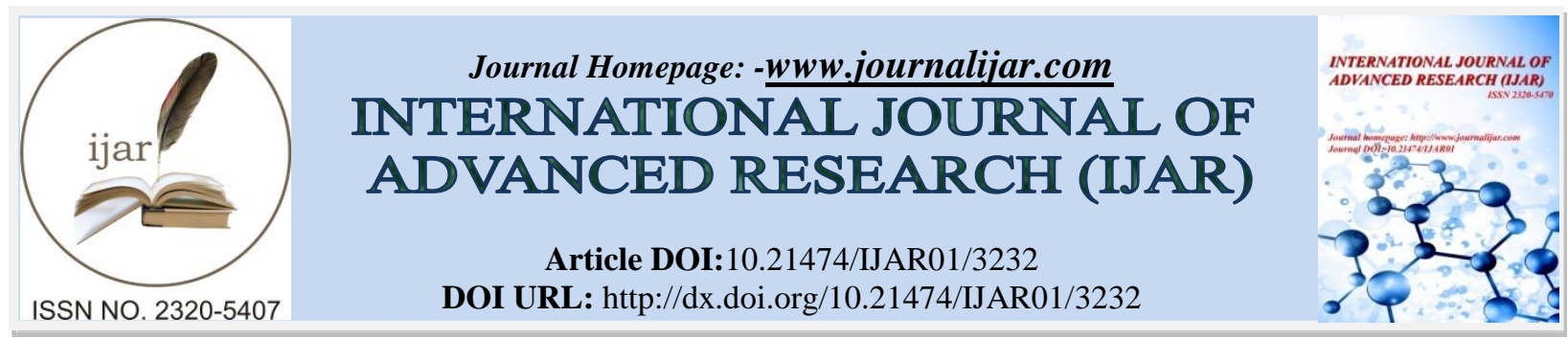

RESEARCH ARTICLE

\title{
A RARE BRAIN TUMOUR- HYPOTHALAMIC PLEOMORPHIC XANTHOASTROCYTOMA IN A 11 YEAR OLD CHILD.
}

Vijaykumar S. Shabadi, Harshil C. Shah.

Department of Neurosurgery, B. J. Medical College and Civil Hospital, Ahmedabad, Gujarat, India 380016.

\section{Manuscript Info}

Manuscript History

Received: 09 December 2016

Final Accepted: 25 January 2017

Published: February 2017

Key words:-

pleomorphicxanthoastrocytoma,

supratentorial,

infratentorial,astrocytomas.

\section{Abstract}

Background:Astrocytomas are the most common glial tumors of the central nervous system among the pediatric age group. Most of the childhood tumors occur infratentorially (medulloblastoma, astrocytoma and ependymomas ). Supratentorialastrocytomas are more likely to be low grade compared with their infratentorial counterpart. Pleomorphic xanthoastrocytomas are rare tumors accounting for only $1 \%$ of all astrocytomas. Despite its highly pleomorphic and bizarre looking cytology, it appeared to have a favorable prognosis and a relatively benign course. Its correct diagnosis is crucial.

Conclusion: Pleomorphic Xanthoastrocytoma is a rare slowly growing tumour of childhood and young adults. Hypothalamus is a very rare site for pleomorphic xanthoastrocytoma. It can present with unusual sensory manifestations. Classical neuroimaging and histopathological characteristic are useful aids in diagnosis. Complete excision is the treatment of choice and gives very good results. A close follow up is always needed, as both recurrence and anaplastic transformation has been reported. Role of radiotherapy and chemotherapy is controversial except in malignant growth.

Copy Right, IJAR, 2017,. All rights reserved.

\section{Introduction:-}

Astrocytomas are the most common glial tumors of the central nervous system among the pediatric age group. Most of the childhood tumors occur infratentorially (medulloblastoma,astrocytoma and ependymomas). Supratentorialastrocytomas are more likely to be low grade compared with their infratentorial counterpart ${ }^{1}$.Pleomorphic xanthoastrocytomas are extremely rare tumours accounting for only $<1 \%$ of all astrocytomas. It was described for the first time by Kepes et al in $1979^{2}$. Among all sites, occurrence of the tumour in hypothalamus is extremely rare. The peculiarity of this tumor is despite its highly pleomorphic and bizarre looking cytology, it appeared to have a favorable prognosis and a relatively benign course. Its correct histological diagnosis is crucial and outcome is good when managed appropriately by surgery. Here we describe one such rare case in a rare locationi.e hypothalamus which was managed successfully by surgery without any adjuvant therapy.

\section{Case Report:-}

A 11 year old female child patient presented in our outpatient department with chief complaints of decreased vision in both eyes and deviation of eyes since 10 days . No history of convulsions, loss of consciousness, vomiting and limb weakness. 
She had normal cognition and behavior. Her school performance was also good. Past and family history was not significant.

On examination:-She had normal higher functions, normal speech, decreased vision in both eyes . Patient had Right eye- Finger Counting +ve and Left eye- Perception of light +veand bilateral $6^{\text {th }}$ nerve paresis. Rest of neurological examination was essentially normal.Hormonal prolife was normal.

MRI BRAIN:- Large suprasellar lesion, which is iso-intense on T1 weighted sequence, iso-intense to hypointense on $\mathrm{T} 2$ weighted sequence and heterogeneously enhancing on post contrast images extending upto floor of $3^{\text {rd }}$ ventricle.

FIG:1
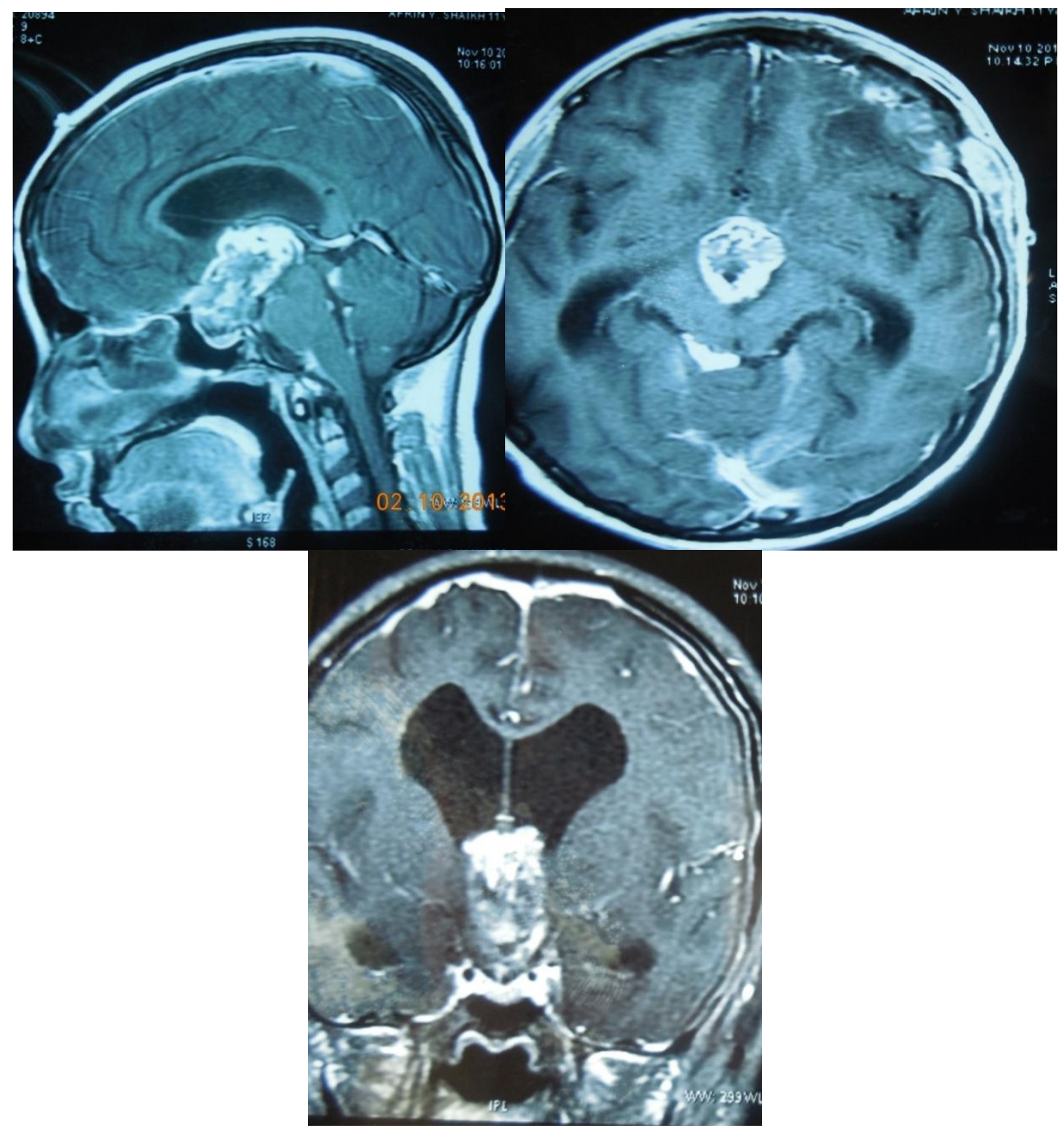

Surgery:- Right side Ventriculoperitoneal shunt was done followed by left frontal craniotomy with sub-frontal approach with near total excision of tumor under GA.

Patient had post operative diabetes insipidus which was managed conservatively. Patient had significant improvement in vision after surgery :- RT eye:- $>6 / 60$ and left eye 6/60. Extra-ocular movements improved significantly and diplopia subsided. 
Histopathology:-S/O Pleomorphic xanthoastrocytoma

FIG:2

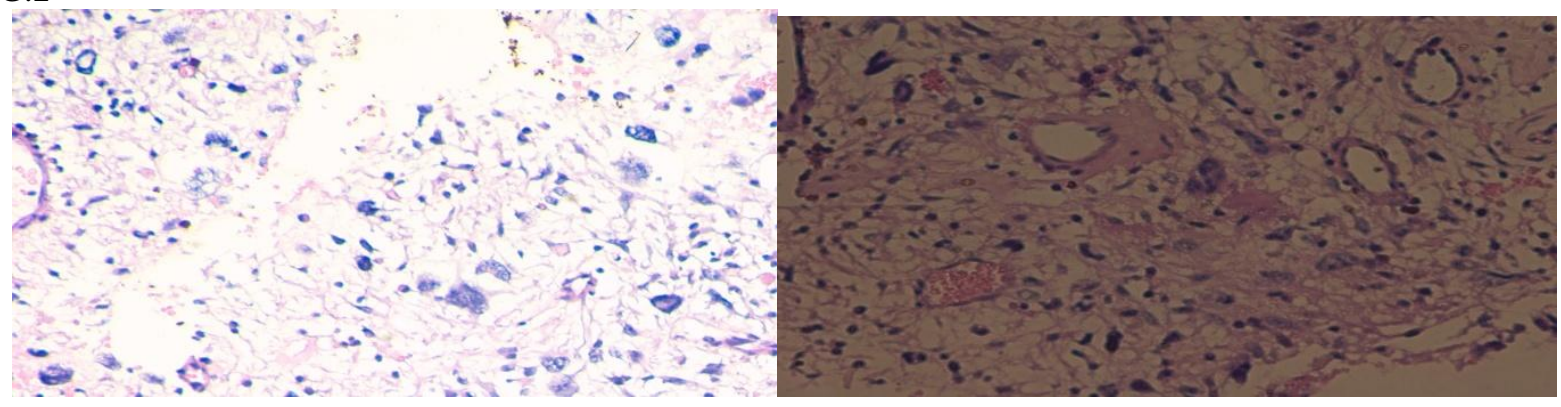

Astrocytic cells having large irregular hyperchromatic nuclei and small lymphocytic like cells.Uni-nucleated and multinucleated tumor giant cells and scattered lymphocytes in a glial background. No significant vascular endothelial proliferation seen. Large pleomorphic cells, prominent eosinophilic granular bodies, lipidized astrocytes, focal perivascular lymphocytes, abundant reticulin network, absent or scant mitoses.

FIG 3: Immediate post op CT:- Complete tumor excision.

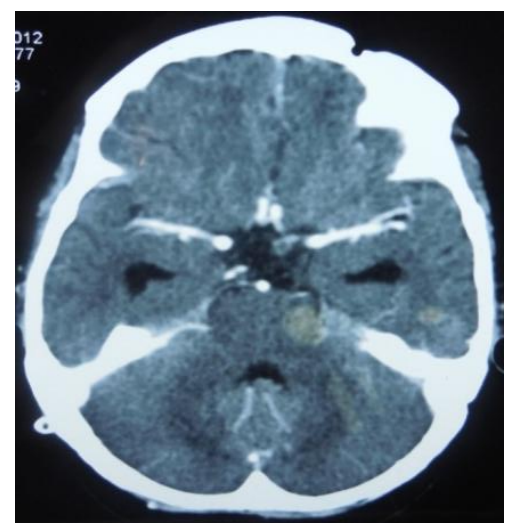

No adjuvant therapy was given. Patient was followed up regularly at 6 months, $1^{\text {st }}$ year and $2^{\text {nd }}$ year.

MRI Brain showed no evidence of tumour at 6 months follow-up. No neurological deficits. Vision improved significantly and diplopia completely subsided. Patient had normal cognitive development.

FIG 4: MRI image at 6 month follow-up.

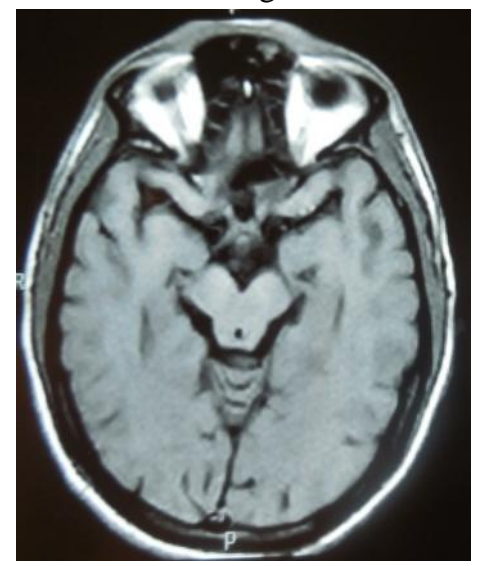

MRI brain after 2 year:- No evidence of tumor recurrence seen after 2 years of follow-up.Patient is attending school regularly with normal intellectual capacity. 
FIG 5: MRI images at $2^{\text {nd }}$ year follow-up:
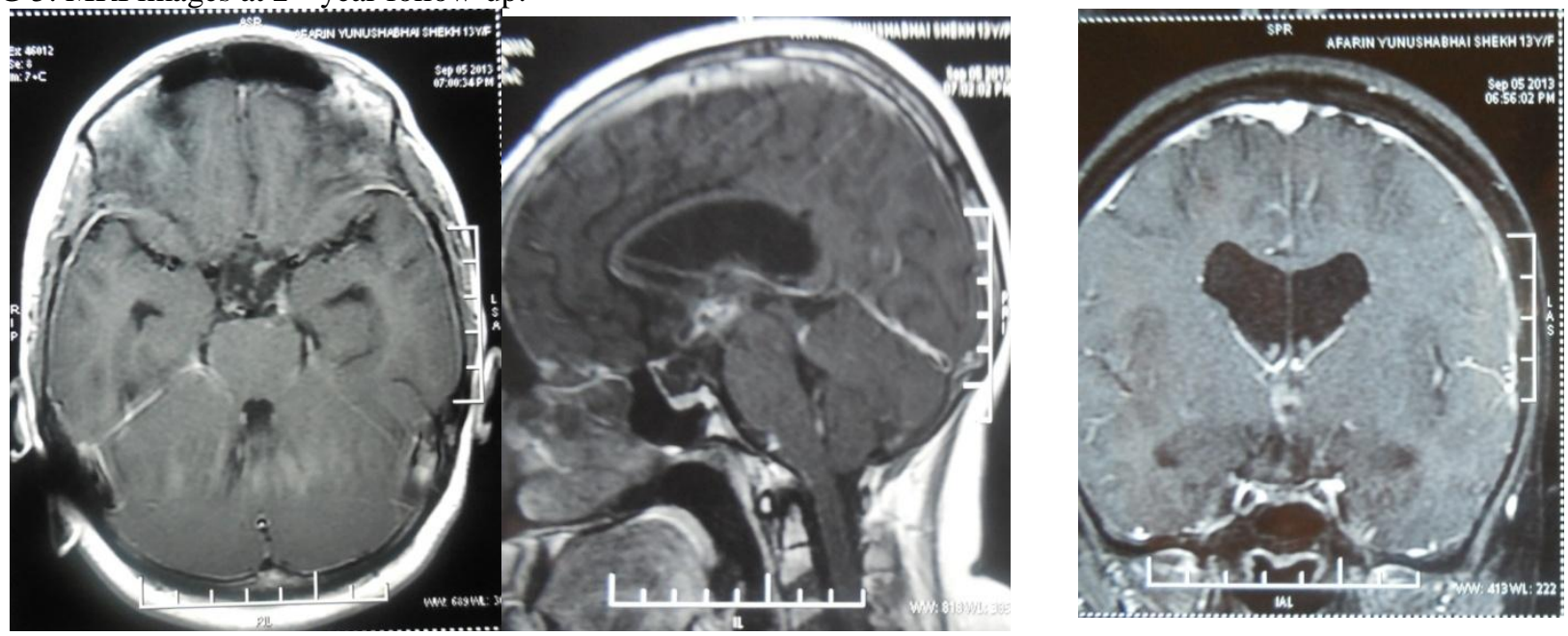

\section{Discussion:-}

In 1993, the World Health Organization recognized Pleomorphic xanthoastrocytoma as a distinct tumor in the classification of grade II tumors. It represents less than $1 \%$ of all the astrocytomas. It can occur throughout the supratentorial compartment, mostly commonly in the temporal lobe, next in frequency are the parietal, occipital and frontal lobes ${ }^{3}$.

To the best of our knowledge, only one case of pleomorphic xanthoastrocytoma involving hypothalamus has been reported in the literature ${ }^{4}$.Location outside the supratentorial compartment has been reported in cerebellum and spinal cord..$^{5}$

Patients typically presents in the second or third decades of life, with the median age of 14 years. Both sexes are affected equally. Most patients have seizures and headache and focal location related deficits ${ }^{2}$. Patient may present with decreased vision if tumor located in sellar and suprasellar region, as in our case.Rarely, patients present with symptomatic hemorrhage from the tumour ${ }^{7}$.

Supratentorialtumours are most often cortically based, adjacent to the meningesbut not involving the dura-mater. On CT and MRI as a cystic structure with an enhancing mural nodule .Both CT and TI weighted imaging reveal a focal, ill defined mass with mixed intensity, isointensity or hypointensity to the gray mater. Well defined contrast enhancement of nodule is often seen on CECT and contrast MRI. On T2 weighted imaging, tumor is hyperintense and cyst also hyperintense. Typically, cyst wall is non enhancing and mild to moderate amount of edema may be present and calcification is very rare $^{2}$.

Grossly, tumors are usually firm, variable in color, and relatively avascular. A yellowish cyst with a mural nodule is common, with the solid portion contacting the lepto-meninges. The characteristic histopathological features of PXA include:- large pleomorphic cells, prominent eosinophilic granular bodies, lipidized astrocytes, focal perivascular lymphocytes, abundant reticulin network, absent or scant mitoses, and absence of necrosis ${ }^{2}$.

PXAs demonstrated immunoreactivity for glial fibrillary acidic protein ( $100 \%)$, S- 100 ( $100 \%)$, class III [beta] tubulin (73\%), synaptophysin (38\%), NF ( nuclear factor) proteins ( 18 and 8\%) and MAP2 ( microtubular associated protein 2$)(8 \%)^{7}$. These tumors are relatively slow clinical course, a favorable prognosis and does not require an aggressive postoperatively therapy. The overall prognosis is good with only $30 \%$ tumors recurring and undergoing anaplastic transformation.

Increased mitotic activity, high MIB-1 and proliferating cell nuclear antigen labeling indices and necrosis are poor prognostic factors, whereas abundant lymphocytic infiltration is associated with more benign biological behavior. Other factors influencing clinical outcome include extent of resection, old age, peri-tumor edema, and increased FDG uptake on PET. A close follow up is needed in order to detect any recurrence with malignant transformation.

Craniotomy and gross total resection is the treatment of choice. Patients with subtotal resection or high mitotic index or necrosis on pathologic examination should at least undergo vigilant, routine gadolinium- enhanced MRI as surveillance for progression. Patients with favorable anatomy, repeat resection may be attempted. Malignant transformation may necessitate adjuvant treatment with chemotherapy and radiation. 


\section{Conclusion:-}

Pleomorphic Xanthoastrocytoma is a very rare slowly growing tumor of childhood and young adults. Hypothalamus is a very rare site for PXA. It can present with unusual sensory manifestations. Classical neuroimaging and histopathological characteristic are useful aids in diagnosis. Complete excision is the treatment of choice, gives very good results. A close follow up is always needed, as both recurrence and anaplastic transformation has been reported. Role of radiotherapy and chemotherapy is controversial except in malignant growth.

\section{References:-}

1. Hanif $\mathrm{G}$ and Shafqat $\mathrm{S}$. Morphological pattern and requency of intracranial tumours in children. J Coll Physicians Surg Pak 2004; 14: 150-2.Youman's textbook of neurosurgery, page no- 1344.

2. Lien RD, Cardenas CA, Rajagopalan S. Pleomorphic Xanthoastrocytoma of the Brain: MR Findings in Six Patients. Am J Roentgenol 1992; 159: 1287-90.

3. Klein $\mathrm{O}$ et al, pleomorphic Xanthoastrocytoma. A review of five observations. Neurochirurgie Nov 2004; 50(5):515:520

4. Hamlat A, Le Strat A, Guegan Y, Ben-Hassel M, Saikali S. Cerebellar pleomorphic xanthoastrocytoma: case report and literature review SurgNeurol 2007; 68: 89-95.

5. Yoshida D et al, A case of pleomorphic Xanthoastrocytoma presenting with massive tumoral hemorrhage. J Neurooncol, 2005 Jan; 71(2): 169-71

6. Giannini CBW Scheithauer BW, Lopes MB, Hirose T, Kros JM, VandenBerg SR. Immunophenotype of Pleomorphic Xanthoastrocytoma. Am J SurgPathol 2002; 26: 479-85. 